\title{
Cecal Volvulus: Rare Presentation of Intestinal Obstruction-Case Report
}

\author{
Muhaned Alhassan, Hatem Al-Saadi \\ Department of General Surgery, Suhar Hospital, Suhar City, Oman \\ Email: hondalhassan@gmail.com
}

How to cite this paper: Alhassan, M. and Al-Saadi, H. (2021) Cecal Volvulus: Rare Presentation of Intestinal Obstruction-Case Report. Case Reports in Clinical Medicine, 10, 359-364.

https://doi.org/10.4236/crcm.2021.1011044

Received: September 10, 2021

Accepted: October 29, 2021

Published: November 1, 2021

Copyright (c) 2021 by author(s) and Scientific Research Publishing Inc. This work is licensed under the Creative Commons Attribution International License (CC BY 4.0).

http://creativecommons.org/licenses/by/4.0/ (c) (i) Open Access

\begin{abstract}
Introduction: Cecal volvulus is a rare cause of intestinal obstruction that occurs $1 \%-1.5 \%$ of all intestinal obstructions. Causes of volvulus are usually unknown but it can be due to a defective peritoneal fixation of the ascending colon and cecum in $10 \%$ and secondary causes (surgical adhesions, colonic carcinoma or diverticulitis). Case Presentation: A 56-year-old woman presented with colicky abdominal pain, bilious vomiting and abdominal distention for two days, who has no history of previous surgery and no other gastrointestinal symptoms or chronic illness. On examinations, she looks ill, dehydrated, abdomen massively distended with exaggerated bowel sound but no signs of peritonitis. CT abdomen with contrast finding: type 11 cecal volvulus seen in midline to the left above the umbilicus reaching $8 \mathrm{~cm}$ with ileocecal junction as well as the elongated appendix is reaching the right iliac fossa. Emergency exploratory laparotomy was done and the finding was, obstructing rectosigmoid tumor with cecal volvulus. Discussion: Cecal volvulus is one of the rare causes of mechanical intestinal obstruction which required urgent surgical intervention, and it occurs due to an axial twist of the caecum, ascending colon and terminal ileum around the mesenteric pedicle. Conclusion: Radiological imaging helps in the diagnosis of cecal volvulus especially CT scan with contrast as gold standard for both diagnosis and assessment for complications. However, this should not delay the time of intervention especially if patient presented at late stage with evidence of peritonitis or bowel ischemia and surgical right hemicolectomy is the most effective treatment option. The colonic pathology always should be assessed, as it could be the primary cause of cecal volvulus.
\end{abstract}

\section{Keywords}

Volvulus, Intestinal Obstruction, Colectomy, Manual Detorsion, Caecopexy, Caecostomy 


\section{Introduction}

Volvulus means that when a loop of intestine twists around itself and the mesentery that supports it resulting in a bowel obstruction. It can also lead to obstruction of the blood supply to the intestine itself, which can result in strangulation and bowel gangrene. It occurs in the organ that contains broad mesentery with a narrow attachment and in relatively mobile GI organs, for example stomach, small bowel, cecum, transverse colon and sigmoid colon. It can occur also in organs other than gastrointestinal tract (ovary, testis). The cecum (10 to 52 percent) and sigmoid colon (43 to 71 percent) are the two most common sites of colonic volvulus [1], and cecal volvulus is a rare cause of intestinal obstruction that occurs $1 \%-1.5 \%$ of all intestinal obstructions [2].

The cecum is the most proximal part of the large intestine, it begins at ileocecal valve (control of passage of small bowel juice into cecum) and can be found in the right iliac fossa of the abdomen and superiorly, the cecum is continuous with the ascending colon. Unlike the ascending colon, the cecum is intraperitoneal and has a variable mesentery. The cecum is derived from the embryologic midgut. Therefore, the vascular supply is via branches of the superior mesenteric vessels.

Causes of volvulus can be a defective peritoneal fixation of the ascending colon and cecum in $10 \%$ of the population [2]-[9]. This fixation permits abnormal mobility of the ascending colon and cecum, making displacement of the right colon into any part of the abdominal cavity possible. Depending on the length of the mobile ascending colon, a variety of obstructive bowel patterns may result. Many authors have described an association with adhesions, membranes, and bands, which may provide a nodal point around which the mobile ascending colon may twist. Although these conditions are frequently present, they are not essential for a volvulus to occur.

Many studies of cecal volvulus have focused on the possibility of volvulus of the right colon occurring in association with obstructive lesions, usually in the left colon [2]-[10]. The most common distal colonic lesions associated with cecal volvulus are colonic carcinoma and diverticulitis.

In the literatures a small number of cases were reported as cecal volvulus causing intestinal obstruction. We are presenting one of the rare causes of mechanical bowel obstruction due to cecal volvulus in a middle aged woman with no previous illness.

\section{Case Presentation}

A 56-year-old woman who was known hypertensive on oral medications presented with colicky abdominal pain, bilious vomiting, abdominal distention and absolute constipation for duration of 2 days.

The condition started with abdominal pain colicky in nature, started at around the umbilicus then radiated to all over her abdomen, increased in severity over the last two days prior to presentation and it's associated with nausea and 
bilious vomiting ( 3 - 4) times per day. Also, there is abdominal distention and absolute constipation for two days. No melena or bleeding per rectum, no history of change in bowel habits, no weight loss or loss of appetite and no history of abdominal operations. No history of previous gastrointestinal pathology.

On examination, she looks ill, dehydrated, in pain, conscious oriented, tachycardic (pulse rate: 112), and low blood pressure (100/60). Oxygen saturation was $100 \%$ on room air and chest examination was clear. Abdomen examination revealed, distended abdomen with full flank, palpable tender mass over the upper abdomen, other abdominal regions were soft, no guarding or rigidity, bowel sound was exaggerated and rectum was empty on digital rectum examination.

Laparotomy investigations revealed, CBC, RFT, LFT, CRP, coagulations profile, bone profiles all were normal. Arterial blood gases showed, PH-7.46, $\mathrm{PCO}_{2}-37 \mathrm{mmHg}, \mathrm{PO}_{2}-102 \mathrm{mmHg}$. $\mathrm{HCO}_{3}-26.30$, lactate- 1 and base excess was 2.5 $\mathrm{mmol} / \mathrm{L}$.

CT abdomen with contrast was done and showed, whole colon is distended with gases, type II cecal volvulus seen in midline to the left above the umbilicus reaching $8 \mathrm{~cm}$ with ileocecal junction as well as the elongated appendix is reaching the right iliac fossa.

Patient was taken to operating theater after adequate and proper resuscitation and anesthesia review and laparotomy was done through the midline vertical incision, the finding was, caecum founded rotated with massive distention of caecum (Figure 1) and slightly distended small bowel, in addition to, obstructed tumor at rectosigmoid junction. detorsion of the caecum and limited right hemicolectomy was done (Figure 2), with diverting ileostomy two drains kept, one at the pelvis and other at the right iliac fossa and abdomen closed in layer. The patient recovered from anesthesia uneventfully and shifted to the word high dependency unit. After the emergency surgery for the acute presentation, the patient was referred to MD team for neoadjuvant chemotherapy for further management in a specialist center.

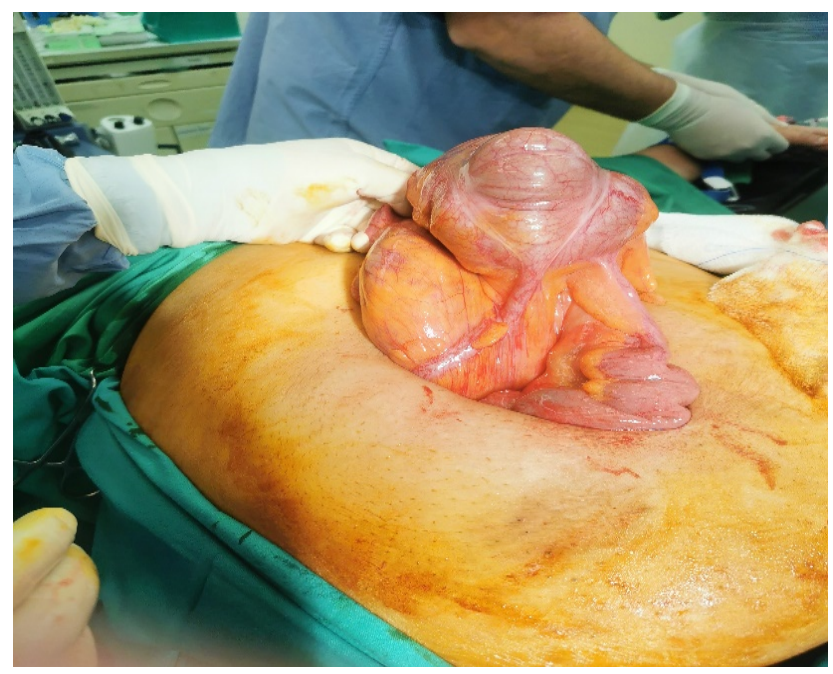

Figure 1. Showed redundant distended mobile cecum. 


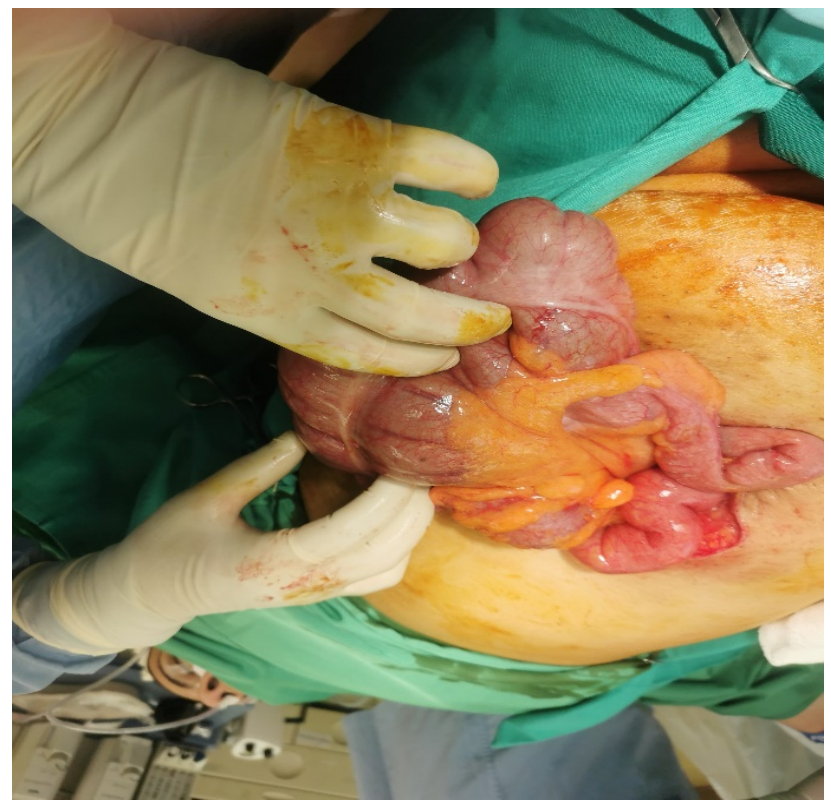

Figure 2. Showed cecum after reduction of torsion along its mesentery.

\section{Discussion}

Cecal volvulus is one of the rare causes of mechanical intestinal obstruction that requires urgent surgical intervention and it occurs due to an axial twist of the caecum, ascending colon and terminal ileum around the mesenteric pedicle [2].

Approximately $1 \%-1.5 \%$ of all cases of intestinal obstructions are caused by cecal volvulus, and $11 \%$ of all volvulus-related intestinal obstruction. Overall incidence is $2.8-7.1$ cases per million each year [3].

Commonly occur at age group between 40 - 60 years old and most commonly occur in female [4] which is same in our case presentation.

In Oman only one case was reported in the literature for young female patient who presented with cecal volvulus and had a history of abdominal hysterectomy before which is going with secondary cause of cecal volvulus (previous operation and adhesions) [5].

Main presentation is cardinal symptoms of intestinal obstruction (colicky abdominal pain, bilious vomiting, abdominal distention and absolute constipation) and may present with all features or some of them and in late presentation they may be presented with symptoms of bowel ischemia or even peritonitis [6].

Diagnosis is usually based on radiological imaging; on plan abdominal X-ray the diagnosis is made if three typical signs are present: caecum dilatation; a single air-fluid level in the right lower quadrant; and absence of gas in the colon but in $30 \%$ of patients these radiographic features are not existed [6]. CT scan is preferred imaging modality for the diagnosis of acute cecal volvulus also in detection of complications (e.g., bowel ischemia) [7]. The three diagnostic CT signs associated with acute cecal volvulus are: coffee bean, bird beak, and whirl signs [7]. 
Several authors have reported successful colonoscopic reduction of cecal volvulus [8]. The success rate for colonoscopic reduction of cecal volvulus is only $30 \%$ and given the potential for colonic perforation colonoscopy is not advised in the management of cecal volvulus.

The most effective treatment is surgical options which include manual detorsion, caecopexy, caecostomy, and colectomy by open or laparoscopic approaches. Given the unusual nature of the disease, there are no prospective treatment trials to guide management decisions [8] and in our patient, the most suitable option for us was colectomy.

\section{Conclusions}

Cecal volvulus is one of the rare causes of acute intestinal obstruction that requires surgical intervention with serious morbidity if diagnosis is delayed, for this reason it needs high index of suspicion for diagnosis.

Causes of cecal volvulus are usually unknown but it can be due to secondary cause. Most commonly occur in females at middle age group.

Radiological imaging helps in the diagnosis especially CT scan with contrast as gold standard for both diagnosis and assessment for complications. However, this should not delay the time of intervention especially if patient presented at late stage with evidence of peritonitis or bowel ischemia and surgical right hemicolectomy is the most effective treatment option.

\section{Conflicts of Interest}

The authors declare no conflicts of interest regarding the publication of this paper.

\section{References}

[1] Delabrousse, E., Sarliève, P., Sailley, N., Aubry, S. and Kastler, B.A. (2007) Cecal Volvulus: CT Findings and Correlation with Pathophysiology. Emergency Radiology, 14, 411. https://doi.org/10.1007/s10140-007-0647-4

[2] Rabinovici, R., Simansky, D.A. and Kaplan, O. (1990) Cecal Volvulus. Diseases of the Colon \& Rectum, 33, 765-769. https://doi.org/10.1007/BF02052323

[3] Bandurski, R., Zareba, K. and Kedra, B. (2011) Cecal Volvulus as a Rare Cause of Intestinal Obstruction. Polski Przeglad Chirurgiczny, 83, 515-517. https://doi.org/10.2478/v10035-011-0080-y

[4] Takada, K., Hamada, Y., Sato, M., et al. (2007) Cecal Volvulus in Children with Mental Disability. Pediatric Surgery International, 23, 1011-1014.

https://doi.org/10.1007/s00383-007-1987-6

[5] Al Mahruqi, G., Al Ebrahim, M., Al Aghbari, S., et al. (2019) Cecal Volvulus: A Case Report \& A Literature Review. International Journal of Innovative Research in Medical Science, 4, 391-394. https://doi.org/10.23958/ijirms/vol04-i06/680

[6] Halabi, W.J., Jafari, M.D., Kang, C.Y., et al. (2014) Colonic volvulus in the United States: Trends, Outcomes, and Predictors of Mortality. Annals of Surgery, 259, 293-301 https://doi.org/10.1097/SLA.0b013e31828c88ac

[7] Delabrousse, E., Sarliève, P., Sailley, N., Aubry, S. and Kastler, B.A. (2007) Cecal- 
volvulus: CT Findings and Correlation with Pathophysiology. Emergency Radiolo$g y$, 14, 411-415. https://doi.org/10.1007/s10140-007-0647-4

[8] Madiba, T.E. and Thomson, S.R. (2002) The Management of Cecal Volvulus. Diseases of the Colon \& Rectum, 45, 264-267.

https://doi.org/10.1007/s10350-004-6158-4

[9] Wolfer, J.A., Beaton, L.E. and Anson, B.J. (1942) Volvulus of the Cecum: Anatomical Factors in its Etiology. Surgery, Gynecology and Obstetrics, 74, 882.

[10] Rivo, M., Farrell, G.E. and Schauffer, I.A. (1957) The Association of Volvulus of the Cecum and Ascending Colon with Obstructive Colonic Lesions. American Journal of Roentgenology, 78, 587-590. 